\title{
The spatially inhomogeneous Hopf bifurcation induced by memory delay in a memory-based diffusion system*
}

\author{
Yongli Song ${ }^{1}$, Yahong Peng ${ }^{2 \dagger}$ Tonghua Zhang ${ }^{3}$ \\ ${ }^{1}$ Department of Mathematics, Hangzhou Normal University, Hangzhou 311121, China \\ ${ }^{2}$ Department of Mathematics, Donghua University, Shanghai, 201620, China \\ ${ }^{3}$ Department of Mathematics, Swinburne University of Technology, Hawthorn, VIC3122, Australia
}

\begin{abstract}
The memory-based diffusion systems have wide applications in practice. Hopf bifurcations are observed from such systems. To meet the demand for computing the normal forms of the Hopf bifurcations of such systems, we develop an effective new algorithm where the memory delay is treated as the perturbation parameter. To illustrate the effectiveness of the algorithm, we consider a diffusive predator-prey system with memory-based diffusion and Holling type-II functional response. By employing this newly developed procedure, we investigate the direction and stability of the delay-induced mode- 1 and mode- 2 Hopf bifurcations. Numerical simulations confirm our theoretical findings, that is the existence of stable spatially inhomogeneous periodic solutions with mode- 1 and mode-2 spatial patterns, and the transition from the unstable mode-2 spatially inhomogeneous periodic solution to the stable mode-1 spatially inhomogeneous periodic solution.
\end{abstract}

Keywords: Spatial memory; delay; Hopf bifurcation; normal form; periodic solution MSC2010: 35B10; 37L10; 37G05

\section{Introduction}

The study of delay-induced Hopf bifurcations is a very active research area in recent years, and has attracted many researchers' attention due to its importance. In the study of nonlinear dynamical systems that may exist rich dynamics, such as Hopf bifurcation, the normal form plays an import role as it can be used to determine the direction and stability of the Hopf bifurcation. Thus, many researchers have dedicated themselves to the development of ways

\footnotetext{
*Partially supported by the Natural Science Foundation of China (Nos.11971143, 12071105), and Natural
} Science Foundation of Zhejiang Province of China (No.LY19A010010).

†Corresponding author, Email: pengyahong@dhu.edu.cn 
calculating normal forms of the nonlinear dynamical systems. For example, Hassard, Kazarinoff and Wan [9] and Faria and Magalhaes [7] proposed an algorithm for computing the normal form of the Hopf bifrucations for delay differential systems (without diffusion), respectively; Faria [6] developed an algorithm for reaction-diffusion systems with time delays, but which only appear in the reaction terms; most recently Song and his colleagues [23, 28] developed some algorithms for reaction-diffusion systems with both time delay and nonlocal spatial average. It is now known that in the classical delay reaction-diffusion systems, the first delay-induced Hopf bifurcation is often homogeneous and the bifurcating periodic oscillation is spatially homogeneous [2, 3, 5, 1015, 17, 21, 26, 27, 29 33. And the aforementioned algorithms were developed for such scenarios.

However, in applications, the delay reaction-diffusion systems may not be homogeneous in space. For example, very recently, to better understand how memory influences the animal movement, Shi et al. [19] proposed a modified Fick's law where in addition to the negative gradient of the density distribution function at the present time, there is a directed movement toward the negative gradient of the density distribution function at past time, and then based on the modified Fick's law, they formulated a single species diffusive model with spatial memory. Since then, there have recently been an increasing activities and interests on the study of the dynamics of the reaction-diffusion equations with spatial memory [1, 16, 18, 20, 22, For this kind of equations, the phenomenon that the first delay-induced Hopf bifurcation is inhomogeneous has been observed by [18, 24]. Based on the assumption that the prey has no memory or cognition, we introduced the spatial memory to the diffusive predator-prey model and proposed the following model with random and memory-based diffusions subject to Neumann boundary conditions on one-dimensional spatial domain $(0, \ell \pi)$ with $\ell \in \mathbb{R}^{+}$is

$$
\left\{\begin{aligned}
\frac{\partial u(x, t)}{\partial t}= & d_{11} u_{x x}(x, t)+f(u(x, t), v(x, t)), & & 0<x<\ell \pi, t>0, \\
\frac{\partial v(x, t)}{\partial t}= & d_{22} v_{x x}(x, t)-d_{21}\left(v(x, t) u_{x}(x, t-\tau)\right)_{x} & & \\
& +g(u(x, t), v(x, t)), & & 0<x<\ell \pi, t>0 \\
u_{x}(0, t)= & u_{x}(\ell \pi, t)=v_{x}(0, t)=v_{x}(\ell \pi, t)=0, & & t \geq 0
\end{aligned}\right.
$$

where $d_{11}$ and $d_{22}$ are the Fickian diffusion coefficients, $d_{12}$ and $d_{21}$ are the memory-based diffusion coefficients, the time delay, denoted by $\tau>0$ represents the averaged memory period of predator. It has been shown that delay-induced first Hopf bifurcations are often spatially inhomogeneous [22].

Compared with the classical reaction-diffusion system with delay, system (1.1) has two main characteristics: $(i)$ the delay appears in the diffusion term; $(i i)$ the diffusion term is not linear. Therefore, algorithms, in the existing literatures such as [6, 9, 23, 28], that were developed for 
analysing the Hopf bifurcation of the reaction-diffusion systems, can not be applied to the system (1.1). To bridge the gap, in this paper, we aim to develop an algorithm that can calculate the normal form for analysing the Hopf bifurcation which may occur in a spatially inhomogeneous scenario and be induced by the memory delay in (1.1). And then we apply this algorithm to a diffusive predator-prey system with memory-based diffusion and Holling type-II functional response. More precisely, we organize the rest of our paper as follows. In Section 2, we derive the algorithm of calculating normal form of spatially Hopf bifurcation induced by memory delay for system (1.1). In Section 3, we study the direction and stability of delay-induced mode-1 and mode-2 Hopf bifurcations in the diffusive predator-prey system with memory-based diffusion and Holling type-II functional response and the numerical simulations are used to illustrate the theoretical results. Finally, we give a brief discussion and conclude our study in Section 4. For the convenience of discussion, throughout the paper, we let $\mathbb{N}$ represent the set of natural numbers, and $\mathbb{N}_{0}=\mathbb{N} \cup\{0\}$ represent the set of all nonnegative integers.

\section{Calculation of the normal form of the Hopf bifurcation}

\subsection{Characteristic equation at the equilibrium and some basic assumptions}

Without loss of generality, assume that $E_{*}=\left(u_{*}, v_{*}\right)$ is a constant coexistence (positive) equilibrium of system (1.1). Then straightforward calculation gives the linearization of (1.1) at $\left(u_{*}, v_{*}\right)$ :

$$
\left(\begin{array}{l}
u_{t}(x, t) \\
v_{t}(x, t)
\end{array}\right)=D_{1}\left(\begin{array}{l}
u_{x x}(x, t) \\
v_{x x}(x, t)
\end{array}\right)+D_{2}\left(\begin{array}{l}
u_{x x}(x, t-\tau) \\
v_{x x}(x, t-\tau)
\end{array}\right)+A\left(\begin{array}{l}
u(x, t) \\
v(x, t)
\end{array}\right)
$$

where

$$
D_{1}=\left(\begin{array}{cc}
d_{11} & 0 \\
0 & d_{22}
\end{array}\right), D_{2}=\left(\begin{array}{cc}
0 & 0 \\
-d_{21} v_{*} & 0
\end{array}\right), A=\left(\begin{array}{cc}
a_{11} & a_{12} \\
a_{21} & a_{22}
\end{array}\right)
$$

and

$$
a_{11}=\frac{\partial f\left(u_{*}, v_{*}\right)}{\partial u}, a_{12}=\frac{\partial f\left(u_{*}, v_{*}\right)}{\partial v}, a_{21}=\frac{\partial g\left(u_{*}, v_{*}\right)}{\partial u}, a_{22}=\frac{\partial g\left(u_{*}, v_{*}\right)}{\partial v} .
$$

Thus, the characteristic equation of (2.1) is

$$
\prod_{n \in \mathbb{N}_{0}} \Gamma_{n}(\lambda)=0
$$

where $\Gamma_{n}(\lambda)=\operatorname{det}\left(\mathcal{M}_{n}(\lambda)\right)$. Notice

$$
\mathcal{M}_{n}(\lambda)=\lambda I_{2}+(n / \ell)^{2} D_{1}+(n / \ell)^{2} e^{-\lambda \tau} D_{2}-A,
$$

with $I_{2}$ is the identity matrix of size 2 . Then we have

$$
\Gamma_{n}(\lambda)=\operatorname{det}\left(\mathcal{M}_{n}(\lambda)\right)=\lambda^{2}-T_{n} \lambda+\widetilde{J}_{n}(\tau)=0,
$$


where

$$
\begin{aligned}
T_{n} & =\operatorname{Tr}(A)-\operatorname{Tr}\left(D_{1}\right)(n / \ell)^{2}, \\
\widetilde{J}_{n}(\tau) & =d_{11} d_{22}(n / \ell)^{4}-\left(d_{11} a_{22}+d_{22} a_{11}+d_{21} v_{*} a_{12} e^{-\lambda \tau}\right)(n / \ell)^{2}+\operatorname{Det}(A),
\end{aligned}
$$

with $\operatorname{Tr}(A)=a_{11}+a_{22}, \operatorname{Tr}\left(D_{1}\right)=d_{11}+d_{22}$ and $\operatorname{Det}(A)=a_{11} a_{22}-a_{12} a_{21}$.

For the Hopf bifurcation, we assume that at $\tau=\tau_{c}$, Eq.2.5 has a pair of purely imaginary roots $\pm i \omega_{n_{c}}, \omega_{n_{c}}>0$ for $n=n_{c} \in \mathbb{N}$ and all other eigenvalues have negative real part. Let $\lambda(\tau)=\alpha(\tau)+i \beta(\tau)$ be a pair of roots of Eq. 2.5 near $\tau=\tau_{c}$ satisfying $\alpha\left(\tau_{c}\right)=0$ and $\beta\left(\tau_{c}\right)=\omega_{n_{c}}$. Then, it is easy to verify the transversality condition

$$
\left.\frac{d \operatorname{Re}(\lambda(\tau))}{d \tau}\right|_{\tau=\tau_{c}} \neq 0
$$

\subsection{Algorithm for computing the normal form}

We first introduce some conventional notations: define the two-dimensional real-valued Sobolev space

$$
\mathscr{X}=\left\{U=\left(U_{1}, U_{2}\right)^{T} \in\left(W^{2,2}(0, \ell \pi)\right)^{2}, \frac{\partial U_{1}}{\partial x}=0, \frac{\partial U_{2}}{\partial x}=0, x=0, \ell \pi\right\},
$$

with the inner product defined by

$$
[U, V]=\int_{0}^{\ell \pi} U^{T} V d x, \text { for } U, V \in \mathscr{X},
$$

and let $\mathscr{C}:=C([-1,0] ; \mathscr{X})$ be the Banach space of continuous mappings from $[-1,0]$ to $\mathscr{X}$.

Then, we take a small perturbation of $\tau_{c}$ by setting $\tau=\tau_{c}+\mu,|\mu| \ll 1$ such that $\mu=0$ corresponds to the Hopf bifurcation value for Eq.11.1. Here, $D_{j}, j=1,2$, are given by 2.2 .

Now shift $E_{*}$ to the origin by setting

$$
U(x, t)=\left(U_{1}(x, t), U_{2}(x, t)\right)^{T}=(u(x, t), v(x, t))^{T}-\left(u_{*}, v_{*}\right)^{T},
$$

normalize the delay by rescaling the time variable $t \rightarrow t / \tau$, and rewrite $U(t)$ for $U(x, t)$ and $U_{t} \in \mathscr{C}$ for $U_{t}(\theta)=U(x, t+\theta),-1 \leq \theta \leq 0$. Then, the system (1.1) becomes the compact form

$$
\frac{d U(t)}{d t}=d(\mu) \Delta\left(U_{t}\right)+L(\mu)\left(U_{t}\right)+F\left(U_{t}, \mu\right)
$$

where for $\varphi=\left(\varphi^{(1)}, \varphi^{(2)}\right)^{T} \in \mathscr{C}, d(\mu) \Delta, L(\mu): \mathscr{C} \rightarrow \mathscr{X}, F: \mathscr{C} \times \mathbb{R}^{2} \rightarrow \mathscr{X}$ are given, respectively, by

$$
d(\mu) \Delta(\varphi)=d_{0} \Delta(\varphi)+F^{d}(\varphi, \mu), L(\mu)(\varphi)=\left(\tau_{c}+\mu\right) A \varphi(0)
$$

and

$$
F(\varphi, \mu)=\left(\tau_{c}+\mu\right)\left(\begin{array}{c}
\left.f\left(\varphi^{(1)}(0)+u_{*}, \varphi^{(2)} 0\right)+v_{*}\right) \\
g\left(\varphi^{(1)}(0)+u_{*}, \varphi^{(2)}(0)+v_{*}\right)
\end{array}\right)-L(\mu)(\varphi)
$$


where

$$
\begin{gathered}
d_{0} \Delta(\varphi)=\tau_{c} D_{1} \varphi_{x x}(0)+\tau_{c} D_{2}^{c} \varphi_{x x}(-1), \\
F^{d}(\varphi, \mu)=-d_{21}\left(\tau_{c}+\mu\right)\left(\begin{array}{c}
0 \\
\varphi_{x}^{(1)}(-1) \varphi_{x}^{(2)}(0)+\varphi_{x x}^{(1)}(-1) \varphi^{(2)}(0)
\end{array}\right) \\
+\mu\left(\begin{array}{c}
d_{11} \varphi_{x x}^{(1)}(0) \\
-d_{21} v_{*} \varphi_{x x}^{(1)}(-1)+d_{22} \varphi_{x x}^{(2)}(0)
\end{array}\right) .
\end{gathered}
$$

In what follows, we assume that $F(\varphi, \mu)$ is $C^{k}, k \geq 3$, smooth with respect to $\varphi$ and $\mu$. Noticing that $\mu$ is the perturbation parameter and treated as a variable in the calculation of normal forms, we denote $L_{0}(\varphi)=\tau_{c} A \varphi(0)$ and rewrite (2.7) as the following

$$
\frac{d U(t)}{d t}=d_{0} \Delta\left(U_{t}\right)+L_{0}\left(U_{t}\right)+\widetilde{F}\left(U_{t}, \mu\right)
$$

by separating the linear terms from the nonlinear terms,

$$
\widetilde{F}(\varphi, \mu)=\mu A \varphi(0)+F(\varphi, \mu)+F^{d}(\varphi, \mu)
$$

Then the characteristic equation for the linearized system of 2.10

$$
\frac{d U(t)}{d t}=d_{0} \Delta\left(U_{t}\right)+L_{0}\left(U_{t}\right)
$$

is

$$
\prod_{n \in \mathbb{N}_{0}} \widetilde{\Gamma}_{n}(\lambda)=0
$$

where $\widetilde{\Gamma}_{n}(\lambda)=\operatorname{det}\left(\widetilde{\mathcal{M}}_{n}(\lambda)\right)$ with

$$
\widetilde{\mathcal{M}}_{n}(\lambda)=\lambda I_{2}+\tau_{c}(n / \ell)^{2} D_{1}+\tau_{c}(n / \ell)^{2} e^{-\lambda} D_{2}-\tau_{c} A .
$$

Comparing (2.14) with 2.4), we know that Eq. (2.13) has a pair of purely imaginary roots $\pm i \omega_{c}$ for $n=n_{c} \in \mathbb{N}$, and all other eigenvalues have negative real parts, where $\omega_{c}=\tau_{c} \omega_{n_{c}}$.

It is well known that the eigenvalue problem

$$
-\gamma^{\prime \prime}=\mu \gamma, \quad x \in(0, \ell \pi) ; \quad \gamma^{\prime}(0)=\gamma^{\prime}(\ell \pi)=0
$$

has eigenvalues $\mu_{n}=(n / \ell)^{2}, n \in \mathbb{N}_{0}$, with the corresponding normalized eigenfunctions

$$
\gamma_{n}(x)=\frac{\cos \left(\frac{n x}{\ell}\right)}{\left\|\cos \left(\frac{n x}{\ell}\right)\right\|_{2,2}}= \begin{cases}\frac{1}{\sqrt{\ell \pi}}, & \text { for } n=0, \\ \frac{\sqrt{2}}{\sqrt{\ell \pi}} \cos \left(\frac{n x}{\ell}\right), & \text { for } n \neq 0 .\end{cases}
$$


Let $\beta_{n}^{(j)}=\gamma_{n}(x) e_{j}, j=1,2$, where $e_{j}$ are the unit coordinate vectors of $\mathbb{R}^{2}$. Then the eigenfunctions $\left\{\beta_{n}^{(j)}\right\}_{n=1}^{\infty}$ form an orthonormal basis for $\mathscr{X}$.

Let $\mathscr{B}_{n}=\operatorname{span}\left\{\left[v(\cdot), \beta_{n}^{(j)}\right] \beta_{n}^{(j)} \mid v \in \mathscr{C}, j=1,2\right\}$. Then it is easy to verify that

$$
L_{0}\left(\mathscr{B}_{n}\right) \subset \operatorname{span}\left\{\beta_{n}^{(1)}, \beta_{n}^{(2)}\right\}, n \in \mathbb{N}_{0} .
$$

Assume that $z_{t}(\theta) \in C=C\left([-1,0], \mathbb{R}^{2}\right)$ and

$$
z_{t}^{T}(\theta)\left(\begin{array}{c}
\beta_{n}^{(1)} \\
\beta_{n}^{(2)}
\end{array}\right) \in \mathscr{B}_{n}
$$

Then, on $\mathscr{B}_{n}$, the linearized equation 2.12 is equivalent to the following functional differential equation (FDE) in $C$ :

$$
\dot{z}(t)=L_{0}^{d}\left(z_{t}(\theta)\right)+L_{0}\left(z_{t}(\theta)\right),
$$

where

$$
L_{0}^{d}\left(z_{t}(\theta)\right)=\tau_{c}\left(\begin{array}{cc}
-d_{11}(n / \ell)^{2} & 0 \\
0 & -d_{22}(n / \ell)^{2}
\end{array}\right) z_{t}(0)+\tau_{c}\left(\begin{array}{cc}
0 & 0 \\
d_{21} v_{*}(n / \ell)^{2} & 0
\end{array}\right) z_{t}(-1) .
$$

The characteristic equation of linear system 2.15 is the same as the one given in 2.13 .

Define $\eta_{n}(\theta) \in B V\left([-1,0], \mathbb{R}^{2}\right)$ such that

$$
\int_{-1}^{0} d \eta_{n}(\theta) \varphi(\theta)=L_{0}^{d}(\varphi(\theta))+L_{0}(\varphi(\theta)), \quad \varphi \in C,
$$

and use the adjoint bilinear form on $C^{*} \times C, C^{*}=C\left([0,1], \mathbb{R}^{2 *}\right)$, where $\mathbb{R}^{2 *}$ is a 2-dimensional space of row vectors, as follows

$$
\langle\psi(s), \varphi(\theta)\rangle_{n}=\psi(0) \varphi(0)-\int_{-1}^{0} \int_{0}^{\theta} \psi(\xi-\theta) d \eta_{n}(\theta) \varphi(\xi) d \xi, \quad \text { for } \psi \in C^{*}, \varphi \in C .
$$

Let $\Lambda=\left\{i \omega_{c},-i \omega_{c}\right\}$. Denote the generalized eigenspace of 2.15 associated with $\Lambda$ by $P$ and the corresponding adjoint space by $P^{*}$. Then, by the adjoint theory of functional differential equation [8], $C$ can be decomposed as $C=P \oplus Q$, where $Q=\left\{\varphi \in C:\langle\psi, \varphi\rangle=0, \forall \psi \in P^{*}\right\}$. Choose the bases $\Phi(\theta)$ and $\Psi(s)$ of $P$ and $P^{*}$, respectively, as follows

$$
\Phi(\theta)=(\phi(\theta), \bar{\phi}(\theta)), \Psi(s)=\operatorname{col}\left(\psi^{T}(s), \bar{\psi}^{T}(s)\right)
$$

such that $\langle\Psi, \Phi\rangle_{n_{c}}=I_{2}$, where

$$
\phi(\theta)=\left(\begin{array}{c}
\phi_{1}(\theta) \\
\phi_{2}(\theta)
\end{array}\right)=\phi e^{i \omega_{c} \theta}, \psi(s)=\left(\begin{array}{c}
\psi_{1}(\theta) \\
\psi_{2}(\theta)
\end{array}\right)=\psi e^{-i \omega_{c} s},
$$


and

$$
\phi=\left(\begin{array}{c}
1 \\
\frac{i \omega_{n_{c}}+\left(n_{c} / \ell\right)^{2} d_{11}-a_{11}}{a_{12}}
\end{array}\right), \quad \psi=\eta\left(\begin{array}{c}
1 \\
\frac{a_{12}}{i \omega_{n_{c}}+\left(n_{c} / \ell\right)^{2} d_{22}-a_{22}}
\end{array}\right),
$$

with

$$
\eta=\frac{i \omega_{n_{c}}+\left(n_{c} / \ell\right)^{2} d_{22}-a_{22}}{i \omega_{n_{c}}+\left(n_{c} / \ell\right)^{2} d_{11}-a_{11}+i \omega_{n_{c}}+\left(n_{c} / \ell\right)^{2} d_{22}-a_{22}+\tau_{c} a_{12} d_{21} v_{*}\left(n_{c} / \ell\right)^{2} e^{-i \omega_{c}}} .
$$

Using the decomposition $C=P \oplus Q$, the phase space $\mathscr{C}$ for $(2.7)$ can be decomposed as

$$
\mathscr{C}=\mathcal{P} \oplus \mathcal{Q}, \mathcal{P}=\operatorname{Im} \pi, \mathcal{Q}=\operatorname{Ker} \pi
$$

where $\pi: \mathscr{C} \rightarrow \mathcal{P}$ is the projection operator defined by

$$
\pi(\phi)=\Phi(\theta)\left\langle\Psi(0),\left(\begin{array}{c}
{\left[\phi(\cdot), \beta_{n_{c}}^{(1)}\right]} \\
{\left[\phi(\cdot), \beta_{n_{c}}^{(2)}\right]}
\end{array}\right)\right\rangle \gamma_{n_{c}}(x)
$$

Following [6] and [25], we define $\mathscr{C}_{0}^{1}=\{\phi \in \mathscr{C}: \dot{\phi} \in \mathscr{C}, \phi(0) \in \operatorname{dom}(d \Delta)\}$ and let

$$
z_{x}=\left(z_{1}(t) \gamma_{n_{c}}(x), z_{2}(t) \gamma_{n_{c}}(x)\right)^{T}, \Phi(\theta)=\left(\phi_{n_{c}}(\theta), \bar{\phi}_{n_{c}}(\theta)\right) \text {. }
$$

For $\varphi(\theta) \in \mathscr{C}_{0}^{1}$, we have the following decomposition

$$
\varphi(\theta)=\Phi(\theta) z_{x}+w, \quad w=\left(w^{(1)}, w^{(2)}\right)^{T} \in \mathscr{C}_{0}^{1} \cap \operatorname{Ker} \pi:=\mathscr{Q}^{1} .
$$

Following the notations in [6], we define

$$
X_{0}(\theta)=\left\{\begin{array}{l}
0, \quad-1 \leq \theta<0 \\
1, \quad \theta=0
\end{array}\right.
$$

and then

$$
\pi\left(X_{0}(\theta) \widetilde{F}_{2}\left(\Phi(\theta) z_{x}, 0\right)\right)=\Phi(\theta) \Psi(0)\left(\begin{array}{l}
{\left[\widetilde{F}_{2}\left(\Phi(\theta) z_{x}, 0\right), \beta_{n_{c}}^{(1)}\right]} \\
{\left[\widetilde{F}_{2}\left(\Phi(\theta) z_{x}, 0\right), \beta_{n_{c}}^{(2)}\right]}
\end{array}\right) \gamma_{n_{c}}(x)
$$

Let $z=\left(z_{1}(t), z_{2}(t)\right)^{T}$. Then system 2.10 can be decomposed as a system of abstract ODEs on $\mathbb{R}^{4} \times \operatorname{Ker} \pi$ :

$$
\left\{\begin{array}{c}
\dot{z}=B z+\Psi(0)\left(\begin{array}{c}
{\left[\widetilde{F}\left(\Phi(\theta) z_{x}+w, \mu\right), \beta_{n_{c}}^{(1)}\right]} \\
{\left[\widetilde{F}\left(\Phi(\theta) z_{x}+w, \mu\right), \beta_{n_{c}}^{(2)}\right]}
\end{array}\right), \\
\dot{w}=A_{\mathcal{Q}^{1}} w+(I-\pi) X_{0}(\theta) \widetilde{F}\left(\Phi(\theta) z_{x}+w, \mu\right),
\end{array}\right.
$$

where $B=\operatorname{diag}\left\{i \omega_{c},-i \omega_{c}\right\}, A_{\mathcal{Q}^{1}}: \mathcal{Q}^{1} \rightarrow \operatorname{Ker} \pi$ is defined by

$$
A_{\mathscr{Q}^{1}} w=\dot{w}+X_{0}(\theta)\left(L_{0}(w)+L_{0}^{d}(w)-\dot{w}(0)\right) .
$$


Consider the formal Taylor expansion

$$
\widetilde{F}(\varphi, \mu)=\sum_{j \geq 2} \frac{1}{j !} \widetilde{F}_{j}(\varphi, \mu), \quad F(\varphi, \mu)=\sum_{j \geq 2} \frac{1}{j !} F_{j}(\varphi, \mu), \quad F^{d}(\varphi, \mu)=\sum_{j \geq 2} \frac{1}{j !} F_{j}^{d}(\varphi, \mu) .
$$

From (2.11), we have

$$
\widetilde{F}_{2}(\varphi, \mu)=2 \mu A \varphi(0)+F_{2}(\varphi, \mu)+F_{2}^{d}(\varphi, \mu)
$$

and

$$
\widetilde{F}_{j}(\varphi, \mu)=F_{j}(\varphi, \mu)+F_{j}^{d}(\varphi, \mu), j=3,4, \cdots .
$$

Then (2.17) is written as

$$
\left\{\begin{array}{l}
\dot{z}=B z+\sum_{j \geq 2} \frac{1}{j !} f_{j}^{1}(z, w, \mu), \\
\dot{w}=A_{\mathscr{Q}^{1}} w+\sum_{j \geq 2} \frac{1}{j !} f_{j}^{2}(z, w, \mu),
\end{array}\right.
$$

where

$$
\begin{gathered}
f_{j}^{1}(z, w, \mu)=\Psi(0)\left(\begin{array}{c}
{\left[\widetilde{F}_{j}\left(\Phi(\theta) z_{x}+w, \mu\right), \beta_{n_{c}}^{(1)}\right]} \\
{\left[\widetilde{F}_{j}\left(\Phi(\theta) z_{x}+w, \mu\right), \beta_{n_{c}}^{(2)}\right]}
\end{array}\right), \\
f_{j}^{2}(z, w, \mu)=(I-\pi) X_{0}(\theta) \widetilde{F}_{j}\left(\Phi(\theta) z_{x}+w, \mu\right) .
\end{gathered}
$$

In terms of the normal form theory of partial functional differential equations [6], after a recursive transformation of variables of the form

$$
(z, w)=(\widetilde{z}, \widetilde{w})+\frac{1}{j !}\left(U_{j}^{1}(\widetilde{z}, \mu), U_{j}^{2}(\widetilde{z}, \mu)(\theta)\right), j \geq 2,
$$

where $z, \widetilde{z} \in \mathbb{R}^{2}, w, \widetilde{w} \in \mathscr{Q}^{1}$ and $U_{j}^{1}: \mathbb{R}^{3} \rightarrow \mathbb{R}^{2}, U_{j}^{2}: \mathbb{R}^{3} \rightarrow \mathscr{Q}^{1}$ are homogeneous polynomials of degree $j$ in $\widetilde{z}$ and $\mu$, the flow on the local center manifold for 2.10 can be written as

$$
\dot{z}=B z+\sum_{j \geq 2} \frac{1}{j !} g_{j}^{1}(z, 0, \mu),
$$

which is the normal form as in the usual sense for ODEs.

Following [6] and [7], we have

$$
g_{2}^{1}(z, 0, \mu)=\operatorname{Proj}_{\operatorname{Ker}\left(M_{2}^{1}\right)} f_{2}^{1}(z, 0, \mu)
$$

and

$$
g_{3}^{1}(z, 0, \mu)=\operatorname{Proj}_{\operatorname{Ker}\left(M_{3}^{1}\right)} \widetilde{f}_{3}^{1}(z, 0, \mu)=\operatorname{Proj}_{S} \widetilde{f}_{3}^{1}(z, 0,0)+O\left(\mu^{2}|z|\right),
$$

where $\widetilde{f}_{3}^{1}(z, 0, \mu)$ is vector, where elements are polynomials of degree 3 in $(z, \mu)$ obtained from 2.22 after performing the change of variables, and is determined by 2.34,

$$
\operatorname{Ker}\left(M_{2}^{1}\right)=\operatorname{Span}\left\{\left(\begin{array}{l}
\mu z_{1} \\
0
\end{array}\right),\left(\begin{array}{l}
0 \\
\mu z_{2}
\end{array}\right)\right\}
$$




$$
\operatorname{Ker}\left(M_{3}^{1}\right)=\operatorname{Span}\left\{\left(\begin{array}{l}
z_{1}^{2} z_{2} \\
0
\end{array}\right),\left(\begin{array}{l}
\mu^{2} z_{1} \\
0
\end{array}\right),\left(\begin{array}{l}
0 \\
z_{1} z_{2}^{2}
\end{array}\right),\left(\begin{array}{l}
0 \\
\mu^{2} z_{2}
\end{array}\right)\right\}
$$

and

$$
S=\operatorname{Span}\left\{\left(\begin{array}{l}
z_{1}^{2} z_{2} \\
0
\end{array}\right),\left(\begin{array}{l}
0 \\
z_{1} z_{2}^{2}
\end{array}\right)\right\} .
$$

For notational convenience, in what follows we let

$$
\mathcal{H}\left(\alpha z_{1}^{q_{1}} z_{2}^{q_{2}} \mu\right)=\left(\begin{array}{c}
\alpha z_{1}^{q_{1}} z_{2}^{q_{2}} \mu \\
\bar{\alpha} z_{1}^{q_{2}} z_{2}^{q_{1}} \mu
\end{array}\right), \alpha \in \mathbb{C} .
$$

We then calculate $\mathbf{g}_{\mathbf{j}}^{\mathbf{1}}(\mathbf{z}, \mathbf{0}, \mu)$.

\subsubsection{Calculation of $\mathrm{g}_{2}^{1}(\mathrm{z}, 0, \mu)$}

From $(2.9)$, we have

$$
F_{2}^{d}(\varphi, \mu)=F_{20}^{d}(\varphi)+\mu F_{21}^{d}(\varphi)
$$

and

$$
F_{3}^{d}(\varphi, \mu)=\mu F_{31}^{d}(\varphi), F_{j}^{d}(\varphi, \mu)=(0,0)^{T}, j=4,5, \cdots
$$

where

$$
\left\{\begin{array}{l}
F_{20}^{d}(\varphi)=-2 d_{21} \tau_{c}\left(\begin{array}{c}
0 \\
\varphi_{x}^{(1)}(-1) \varphi_{x}^{(2)}(0)+\varphi_{x x}^{(1)}(-1) \varphi^{(2)}(0)
\end{array}\right), \\
F_{21}^{d}(\varphi)=2 D_{1} \varphi_{x x}(0)+2 D_{2} \varphi_{x x}(-1), \\
F_{31}^{d}(\varphi)=-6 d_{21}\left(\begin{array}{c}
0 \\
\varphi_{x}^{(1)}(-1) \varphi_{x}^{(2)}(0)+\varphi_{x x}^{(1)}(-1) \varphi^{(2)}(0)
\end{array}\right),
\end{array}\right.
$$

It is easy to verify that

$$
\begin{gathered}
\left(\begin{array}{c}
{\left[2 \mu A\left(\Phi(0) z_{x}\right), \beta_{n_{c}}^{(1)}\right]} \\
{\left[2 \mu A\left(\Phi(0) z_{x}\right), \beta_{n_{c}}^{(2)}\right]}
\end{array}\right)=2 \mu A\left(\Phi(0)\left(\begin{array}{c}
z_{1} \\
z_{2}
\end{array}\right)\right), \\
\left(\begin{array}{c}
{\left[\mu F_{21}^{d}\left(\Phi(\theta) z_{x}\right), \beta_{n_{c}}^{(1)}\right]} \\
{\left[\mu F_{21}^{d}\left(\Phi(\theta) z_{x}\right), \beta_{n_{c}}^{(2)}\right]}
\end{array}\right) \\
=-2\left(n_{c} / \ell\right)^{2} \mu\left(D_{1}\left(\Phi(0)\left(\begin{array}{l}
z_{1} \\
z_{2}
\end{array}\right)\right)+D_{2}\left(\Phi(-1)\left(\begin{array}{l}
z_{1} \\
z_{2}
\end{array}\right)\right)\right) .
\end{gathered}
$$

In addition, from (2.8), we have for all $\mu \in \mathbb{R}$,

$$
F_{2}\left(\Phi(\theta) z_{x}, \mu\right)=F_{2}\left(\Phi(\theta) z_{x}, 0\right)
$$


It follows from $(2.20)$ that

$$
f_{2}^{1}(z, 0, \mu)=\Psi(0)\left(\begin{array}{l}
{\left[\widetilde{F}_{2}\left(\Phi(\theta) z_{x}, \mu\right), \beta_{n_{c}}^{(1)}\right]} \\
{\left[\widetilde{F}_{2}\left(\Phi(\theta) z_{x}, \mu\right), \beta_{n_{c}}^{(2)}\right]}
\end{array}\right) .
$$

This, together with (2.18), 2.25), 2.28) and (2.29), yields to

$$
g_{2}^{1}(z, 0, \mu)=\operatorname{Proj}_{\operatorname{Ker}\left(M_{2}^{1}\right)} f_{2}^{1}(z, 0, \mu)=\mathcal{H}\left(B_{1} \mu z_{1}\right)
$$

where

$$
B_{1}=2 \psi^{T}\left(A \phi(0)-\left(n_{c} / \ell\right)^{2}\left(D_{1} \phi(0)+D_{2} \phi(-1)\right)\right)=2 i \omega_{n_{c}} \psi^{T} \phi
$$

\subsubsection{Calculation of $\mathrm{g}_{3}^{1}(\mathrm{z}, 0, \mu)$}

In this subsection, we calculate the third term $g_{3}^{1}(z, 0,0)$ in terms of 2.24$)$. Notice that $\frac{1}{3 !} \widetilde{f}_{3}^{1}$ in (2.24) is the term of order 3 obtained after the changes of variables in previous step. Denote

$$
\begin{gathered}
f_{2}^{(1,1)}(z, w, 0)=\Psi(0)\left(\begin{array}{l}
{\left[F_{2}\left(\Phi(\theta) z_{x}+w, 0\right), \beta_{n_{c}}^{(1)}\right]} \\
{\left[F_{2}\left(\Phi(\theta) z_{x}+w, 0\right), \beta_{n_{c}}^{(2)}\right]}
\end{array}\right), \\
f_{2}^{(1,2)}(z, w, 0)=\Psi(0)\left(\begin{array}{l}
{\left[F_{2}^{d}\left(\Phi(\theta) z_{x}+w, 0\right), \beta_{n_{c}}^{(1)}\right]} \\
{\left[F_{2}^{d}\left(\Phi(\theta) z_{x}+w, 0\right), \beta_{n_{c}}^{(2)}\right]}
\end{array}\right) .
\end{gathered}
$$

In addition, it follows from 2.31) that $g_{2}^{1}(z, 0,0)=(0,0)^{T}$. Then $\widetilde{f}_{3}^{1}(z, 0,0)$ is determined by

$$
\begin{aligned}
& \widetilde{f}_{3}^{1}(z, 0,0) \\
= & f_{3}^{1}(z, 0,0)+\frac{3}{2}\left[\left(D_{z} f_{2}^{1}(z, 0,0)\right) U_{2}^{1}(z, 0)+\left(D_{w} f_{2}^{(1,1)}(z, 0,0)\right) U_{2}^{2}(z, 0)(\theta)\right. \\
& \left.+\left(D_{w, w_{x}, w_{x x}} f_{2}^{(1,2)}(z, 0,0)\right) U_{2}^{(2, d)}(z, 0)(\theta)\right],
\end{aligned}
$$

where $f_{2}^{1}(z, 0,0)=f_{2}^{(1,1)}(z, 0,0)+f_{2}^{(1,2)}(z, 0,0)$,

$$
\begin{aligned}
& D_{w, w_{x}, w_{x x}} f_{2}^{(1,2)}(z, 0,0)=\left(D_{w} f_{2}^{(1,2)}(z, 0,0), D_{w_{x}} f_{2}^{(1,2)}(z, 0,0), D_{w_{x x}} f_{2}^{(1,2)}(z, 0,0)\right), \\
& U_{2}^{1}(z, 0)=\left(M_{2}^{1}\right)^{-1} \operatorname{Proj}_{I m\left(M_{2}^{1}\right)} f_{2}^{1}(z, 0,0), \quad U_{2}^{2}(z, 0)(\theta)=\left(M_{2}^{2}\right)^{-1} f_{2}^{2}(z, 0,0),
\end{aligned}
$$

and

$$
U_{2}^{(2, d)}(z, 0)(\theta)=\operatorname{col}\left(U_{2}^{2}(z, 0)(\theta), U_{2 x}^{2}(z, 0)(\theta), U_{2 x x}^{2}(z, 0)(\theta)\right) .
$$

Next, we compute $\operatorname{Proj}_{S} \widetilde{f}_{3}^{1}(z, 0,0)$ step by step according to 2.34). The calculation is divided into the following four steps. 


\section{Step 1: The calculation of $\operatorname{Proj}_{S} f_{3}^{1}(z, 0,0)$}

Let

$$
F_{3}\left(\Phi(\theta) z_{x}, 0\right)=\gamma_{n_{c}}^{3}(x)\left(\sum_{q_{1}+q_{2}=3} A_{q_{1} q_{2}} z_{1}^{q_{1}} z_{2}^{q_{2}}\right), q_{1}, q_{2} \in \mathbb{N}_{0} .
$$

From $(2.19)$ and $(2.26)$, we have $\widetilde{F}_{3}\left(\Phi(\theta) z_{x}, 0\right)=F_{3}\left(\Phi(\theta) z_{x}, 0\right)$. Then it follows from 2.20) and (2.37) that

$$
f_{3}^{1}(z, 0,0)=\Psi(0)\left(\sum_{q_{1}+q_{2}=3} A_{q_{1} q_{2}} \int_{0}^{\ell \pi} \gamma_{n_{c}}^{q_{1}+q_{2}+1}(x) d x z_{1}^{q_{1}} z_{2}^{q_{2}}\right),
$$

which, together with the fact that $\int_{0}^{\ell \pi} \gamma_{n_{c}}^{4}(x) d x=\frac{3}{2 \ell \pi}$, implies that

$$
\operatorname{Proj}_{S} f_{3}^{1}(z, 0,0)=\mathcal{H}\left(B_{21} z_{1}^{2} z_{2}\right)
$$

where

$$
B_{21}=\frac{3}{2 \ell \pi} \psi^{T} A_{21}
$$

Step 2: The calculation of $\operatorname{Proj}_{\mathbf{S}}\left(\left(\mathbf{D}_{\mathbf{z}} \mathbf{f}_{\mathbf{2}}^{\mathbf{1}}\right)(\mathbf{z}, \mathbf{0}, \mathbf{0}) \mathbf{U}_{\mathbf{2}}^{\mathbf{1}}(\mathbf{z}, \mathbf{0})\right)$

Form 2.18 and 2.25, we have

$$
\widetilde{F}_{2}\left(\Phi(\theta) z_{x}, 0\right)=F_{2}\left(\Phi(\theta) z_{x}, 0\right)+F_{20}^{d}\left(\Phi(\theta) z_{x}\right)
$$

By (2.8), we write

$$
\begin{aligned}
& F_{2}\left(\Phi(\theta) z_{x}+w, \mu\right)=F_{2}\left(\Phi(\theta) z_{x}+w, 0\right) \\
= & \gamma_{n_{c}}^{2}(x)\left(\sum_{q_{1}+q_{2}=2} A_{q_{1} q_{2}} z_{1}^{q_{1}} z_{2}^{q_{2}}\right)+\mathcal{S}_{2}\left(\Phi(\theta) z_{x}, w\right)+O\left(|w|^{2}\right), q_{1}, q_{2} \in \mathbb{N}_{0},
\end{aligned}
$$

where $\mathcal{S}_{2}\left(\Phi(\theta) z_{x}, w\right)$ is the second cross terms of $\Phi(\theta) z_{x}$ and $w$. In addition, by (2.25) and (2.27), we write

$$
\begin{aligned}
& F_{2}^{d}\left(\Phi(\theta) z_{x}, 0\right)=F_{20}^{d}\left(\Phi(\theta) z_{x}\right) \\
= & \left(n_{c} / \ell\right)^{2}\left(\xi_{n_{c}}^{2}(x)-\gamma_{n_{c}}^{2}(x)\right)\left(\sum_{q_{1}+q_{2}=2} A_{q_{1} q_{2}}^{d} z_{1}^{q_{1}} z_{2}^{q_{2}}\right),
\end{aligned}
$$

where

$$
\begin{gathered}
\xi_{n_{c}}(x)=\frac{\sqrt{2}}{\sqrt{\ell \pi}} \sin \left(\frac{n_{c} x}{\ell}\right), \\
A_{20}^{d}=-2 d_{21} \tau_{c}\left(\begin{array}{c}
0 \\
\phi_{1}(-1) \phi_{2}(0)
\end{array}\right)=\overline{A_{02}^{d}} \\
0 \\
A_{11}^{d}=-2 d_{21} \tau_{c}\left(\begin{array}{c}
2 \\
2 \operatorname{Re}\left\{\phi_{1}(-1) \overline{\phi_{2}}(0)\right\}
\end{array}\right) .
\end{gathered}
$$


It is easy to verify that

$$
\int_{0}^{\ell \pi} \gamma_{n_{c}}^{3}(x) d x=\int_{0}^{\ell \pi} \xi_{n_{2}}^{2}(x) \gamma_{n_{c}}(x) d x=0
$$

Then, by 2.40)-2.42, we have

$$
f_{2}^{1}(z, 0,0)=\Psi(0)\left(\begin{array}{l}
{\left[\widetilde{F}_{2}\left(\Phi(\theta) z_{x}, 0\right), \beta_{n_{c}}^{(1)}\right]} \\
{\left[\widetilde{F}_{2}\left(\Phi(\theta) z_{x}, 0\right), \beta_{n_{c}}^{(2)}\right]}
\end{array}\right)=\left(\begin{array}{c}
0 \\
0
\end{array}\right)
$$

Hence,

$$
\operatorname{Proj}_{S}\left[\left(D_{z} f_{2}^{1}\right)(z, 0,0) U_{2}^{1}(z, 0)\right]=\left(\begin{array}{c}
0 \\
0
\end{array}\right)
$$

Step 3: The calculation of $\operatorname{Proj}_{\mathbf{S}}\left(\left(\mathbf{D}_{\mathrm{w}} \mathbf{f}_{\mathbf{2}}^{(\mathbf{1 , 1})}(\mathrm{z}, \mathbf{0}, \mathbf{0})\right) \mathbf{U}_{\mathbf{2}}^{\mathbf{2}}(\mathbf{z}, \mathbf{0})(\theta)\right)$

Let

$$
U_{2}^{2}(z, 0)(\theta) \triangleq h(\theta, z)=\sum_{n \in \mathbb{N}_{0}} h_{n}(\theta, z) \gamma_{n}(x)
$$

where

$$
h_{n}(\theta, z)=\sum_{q_{1}+q_{2}=2} h_{n, q_{1} q_{2}}(\theta) z_{1}^{q_{1}} z_{2}^{q_{2}} .
$$

Then, from 2.32 and 2.45$)$, we obtain

$$
\begin{aligned}
\left(D_{w} f_{2}^{(1,1)}(z, 0,0)\right) U_{2}^{2}(z, 0)(\theta) & \left(\begin{array}{l}
{\left[\left.D_{w} F_{2}\left(\Phi(\theta) z_{x}+w, 0\right)\right|_{w=0}\left(\sum_{n \in \mathbb{N}_{0}} h_{n}(\theta, z) \gamma_{n}(x)\right), \beta_{n_{c}}^{(1)}\right]} \\
=\Psi(0) \\
{\left[\left.D_{w} F_{2}\left(\Phi(\theta) z_{x}+w, 0\right)\right|_{w=0}\left(\sum_{n \in \mathbb{N}_{0}} h_{n}(\theta, z) \gamma_{n}(x)\right), \beta_{n_{c}}^{(2)}\right]}
\end{array}\right) .
\end{aligned}
$$

By 2.41, we obtain

$$
\left.D_{w} F_{2}\left(\Phi(\theta) z_{x}+w, 0\right)\right|_{w=0}\left(\sum_{n \in \mathbb{N}_{0}} h_{n}(\theta, z) \gamma_{n}(x)\right)=\mathcal{S}_{2}\left(\Phi(\theta) z_{x}, \sum_{n \in \mathbb{N}_{0}} h_{n}(\theta, z) \gamma_{n}(x)\right)
$$

and

$$
\begin{aligned}
& \left(\begin{array}{c}
{\left[\mathcal{S}_{2}\left(\Phi(\theta) z_{x}, \sum_{n \in \mathbb{N}_{0}} h_{n}(\theta, z) \gamma_{n}(x)\right), \beta_{n_{c}}^{(1)}\right]} \\
{\left[\mathcal{S}_{2}\left(\Phi(\theta) z_{x}, \sum_{n \in \mathbb{N}_{0}} h_{n}(\theta, z) \gamma_{n}(x)\right), \beta_{n_{c}}^{(2)}\right]}
\end{array}\right) \\
= & \sum_{n \in \mathbb{N}_{0}} b_{n}\left(\mathcal{S}_{2}\left(\phi(\theta) z_{1}, h_{n}(\theta, z)\right)+\mathcal{S}_{2}\left(\bar{\phi}(\theta) z_{2}, h_{n}(\theta, z)\right)\right),
\end{aligned}
$$


where

$$
b_{n}=\int_{0}^{\ell \pi} \gamma_{n_{c}}^{2}(x) \gamma_{n}(x) d x= \begin{cases}\frac{1}{\sqrt{\ell \pi}}, & n=0 \\ \frac{1}{\sqrt{2 \ell \pi}}, & n=2 n_{c} \\ 0, & \text { otherwise }\end{cases}
$$

Hence,

$$
\begin{aligned}
& \left(D_{w} f_{2}^{(1,1)}(z, 0,0)\right) U_{2}^{2}(z, 0)(\theta) \\
= & \Psi(0)\left(\sum_{n=0,2 n_{c}} b_{n}\left(\mathcal{S}_{2}\left(\phi(\theta) z_{1}, h_{n}(\theta, z)\right)+\mathcal{S}_{2}\left(\bar{\phi}(\theta) z_{2}, h_{n}(\theta, z)\right)\right)\right) .
\end{aligned}
$$

Then, we have

$$
\operatorname{Proj}_{S}\left(D_{w} f_{2}^{(1,1)}(z, 0,0) U_{2}^{2}(z, 0)(\theta)\right)=\mathcal{H}\left(B_{22} z_{1}^{2} z_{2}\right)
$$

where

$$
\begin{aligned}
B_{22}= & \frac{1}{\sqrt{\ell \pi}} \psi^{T}\left(\mathcal{S}_{2}\left(\phi(\theta), h_{0,11}(\theta)\right)+\mathcal{S}_{2}\left(\bar{\phi}(\theta), h_{0,20}(\theta)\right)\right) \\
& +\frac{1}{\sqrt{2 \ell \pi}} \psi^{T}\left(\mathcal{S}_{2}\left(\phi(\theta), h_{2 n_{c}, 11}(\theta)\right)+\mathcal{S}_{2}\left(\bar{\phi}(\theta), h_{2 n_{c}, 20}(\theta)\right)\right) .
\end{aligned}
$$

Step 4: The calculation of $\operatorname{Proj}_{\mathbf{S}}\left(\left(\mathrm{D}_{\mathrm{w}, \mathbf{w}_{\mathbf{x}}, \mathbf{w}_{\mathbf{x} x}} \mathbf{f}_{2}^{(1,2)}(\mathrm{z}, \mathbf{0}, \mathbf{0})\right) \mathbf{U}_{2}^{(2, d)}(\mathbf{z}, 0)(\theta)\right)$

Denote $\varphi(\theta)=\left(\varphi^{(1)}, \varphi^{(2)}\right)^{T}=\Phi(\theta) z_{x}$ and

$$
\begin{gathered}
F_{2}^{d}\left(\varphi(\theta), w, w_{x}, w_{x x}\right)=F_{2}^{d}(\varphi(\theta)+w, 0)=F_{20}^{d}(\varphi(\theta)+w) \\
=-2 d_{21} \tau_{c}\left(\begin{array}{c}
0 \\
\left(\varphi_{x x}^{(1)}(-1)+w_{x x}^{(1)}(-1)\right)\left(\varphi^{(2)}(0)+w^{(2)}(0)\right)
\end{array}\right) \\
-2 d_{21} \tau_{c}\left(\begin{array}{c}
0 \\
\left(\varphi_{x}^{(1)}(-1)+w_{x}^{(1)}(-1)\right)\left(\varphi_{x}^{(2)}(0)+w_{x}^{(2)}(0)\right)
\end{array}\right),
\end{gathered}
$$

and for $\phi(\theta)=\left(\phi_{1}(\theta), \phi_{2}(\theta)\right)^{T}, y(\theta)=\left(y_{1}(\theta), y_{2}(\theta)\right)^{T} \in C\left([-1,0], \mathbb{R}^{2}\right)$,

$$
\left\{\begin{array}{l}
\mathcal{S}_{2}^{(d, 1)}(\phi(\theta), y(\theta))=-2 d_{21} \tau_{c}\left(\begin{array}{c}
0 \\
\phi_{1}(-1) y_{2}(0)
\end{array}\right) \\
\mathcal{S}_{2}^{(d, 2)}(\phi(\theta), y(\theta))=-2 d_{21} \tau_{c}\left(\begin{array}{c}
0 \\
\phi_{1}(-1) y_{2}(0)
\end{array}\right)-2 d_{21} \tau_{c}\left(\begin{array}{c}
0 \\
\phi_{2}(0) y_{1}(-1)
\end{array}\right) \\
\mathcal{S}_{2}^{(d, 3)}(\phi(\theta), y(\theta))=-2 d_{21} \tau_{c}\left(\begin{array}{c}
0 \\
\phi_{2}(0) y_{1}(-1)
\end{array}\right)
\end{array}\right.
$$


Then, from (2.33), 2.36 and 2.45), we have

$$
\begin{aligned}
& \left(D_{w, w_{x}, w_{x x}} f_{2}^{(1,2)}(z, 0,0)\right) U_{2}^{(2, d)}(z, 0)(\theta) \\
= & \Psi(0)\left(\begin{array}{c}
{\left[D_{w, w_{x}, w_{x x}} F_{2}^{d}\left(\varphi(\theta), w, w_{x}, w_{x x}\right) U_{2}^{(2, d)}(z, 0)(\theta), \beta_{n_{c}}^{(1)}\right]} \\
{\left[D_{w, w_{x}, w_{x x}} F_{2}^{d}\left(\varphi(\theta), w, w_{x}, w_{x x}\right) U_{2}^{(2, d)}(z, 0)(\theta), \beta_{n_{c}}^{(2)}\right]}
\end{array}\right)
\end{aligned}
$$

and then we obtain

$$
\operatorname{Proj}_{S}\left(\left(D_{w, w_{x}, w_{x x}} f_{2}^{(1,2)}(z, 0,0)\right) U_{2}^{(2, d)}(z, 0)(\theta)\right)=\mathcal{H}\left(B_{23} z_{1}^{2} z_{2}\right)
$$

where

$$
\begin{aligned}
B_{23}= & -\frac{1}{\sqrt{\ell \pi}}\left(n_{c} / \ell\right)^{2} \psi^{T}\left(\mathcal{S}_{2}^{(d, 1)}\left(\phi(\theta), h_{0,11}(\theta)\right)+\mathcal{S}_{2}^{(d, 1)}\left(\bar{\phi}(\theta), h_{0,20}(\theta)\right)\right) \\
& +\frac{1}{\sqrt{2 \ell \pi}} \psi^{T} \sum_{j=1,2,3} b_{2 n_{c}}^{(j)}\left(\mathcal{S}_{2}^{(d, j)}\left(\phi(\theta), h_{2 n_{c}, 11}(\theta)\right)+\mathcal{S}_{2}^{(d, j)}\left(\bar{\phi}(\theta), h_{2 n_{c}, 20}(\theta)\right)\right)
\end{aligned}
$$

with

$$
b_{2 n_{c}}^{(1)}=-\left(n_{c} / \ell\right)^{2}, b_{2 n_{c}}^{(2)}=2\left(n_{c} / \ell\right)^{2}, b_{2 n_{c}}^{(3)}=-\left(2 n_{c} / \ell\right)^{2} .
$$

\subsubsection{Normal form of the Hopf bifurcation and the corresponding coefficients}

According to the above calculations, we have obtained the normal form of the Hopf bifurcation in the following form

$$
\dot{z}=B z+\frac{1}{2}\left(\begin{array}{c}
B_{1} z_{1} \mu \\
\bar{B}_{1} z_{2} \mu
\end{array}\right)+\frac{1}{3 !}\left(\begin{array}{c}
B_{2} z_{1}^{2} z_{2} \\
\bar{B}_{2} z_{1} z_{2}^{2}
\end{array}\right)+O\left(|z| \mu^{2}+\left|z^{4}\right|\right),
$$

where

$$
B_{1}=2 i \omega_{n_{c}} \psi^{T} \phi, B_{2}=B_{21}+\frac{3}{2}\left(B_{22}+B_{23}\right),
$$

and $B_{2 j}, j=1,2,3$, are determined by 2.39$), 2.48$ and 2.51). Through the change of variables $z_{1}=w_{1}-i w_{2}, z_{2}=w_{1}+i w_{2}$ and $w_{1}=\rho \cos \xi, w_{2}=\rho \sin \xi$, the normal form 2.52 can be written as the following form in polar coordinates

$$
\dot{\rho}=K_{1} \mu \rho+K_{2} \rho^{3}+O\left(\mu^{2} \rho+|(\rho, \mu)|^{4}\right),
$$

with $K_{1}=\frac{1}{2} \operatorname{Re}\left(B_{1}\right), K_{2}=\frac{1}{3 !} \operatorname{Re}\left(B_{2}\right)$. According to [4], the sign of $K_{1} K_{2}$ determines the direction of the bifurcation (supercritical for $K_{1} K_{2}<0$ and subcritical for $K_{1} K_{2}>0$ ), and the sign of $K_{2}$ determines the stability of the Hopf bifurcating periodic orbits (stable for $K_{2}<0$ and unstable for $K_{2}>0$ ).

From (2.39), 2.48) and (2.51), it is obvious that in order to obtain the value of $K_{2}$, we still need to compute $h_{0,20}(\theta), h_{0,11}(\theta), h_{2 n_{c}, 20}(\theta), h_{2 n_{c}, 11}(\theta)$, and $A_{i j}$. 
From [6], we have

$$
\begin{aligned}
& M_{2}^{2}\left(h_{n}(\theta, z) \gamma_{n}(x)\right) \\
= & D_{z}\left(h_{n}(\theta, z) \gamma_{n}(x)\right) B z-A_{\mathcal{Q}^{1}}\left(h_{n}(\theta, z) \gamma_{n}(x)\right),
\end{aligned}
$$

which leads to

$$
\begin{aligned}
& \left(\begin{array}{l}
{\left[M_{2}^{2}\left(h_{n}(\theta, z) \gamma_{n}(x)\right), \beta_{n}^{(1)}\right]} \\
{\left[M_{2}^{2}\left(h_{n}(\theta, z) \gamma_{n}(x)\right), \beta_{n}^{(2)}\right]}
\end{array}\right) \\
= & 2 i \omega_{c}\left(h_{n, 20}(\theta) z_{1}^{2}-h_{n, 02}(\theta) z_{2}^{2}\right) \\
& -\left(\dot{h}_{n}(\theta, z)+X_{0}(\theta)\left(\mathscr{L}_{0}\left(h_{n}(\theta, z)\right)-\dot{h}_{n}(0, z)\right)\right),
\end{aligned}
$$

where

$$
\mathscr{L}_{0}\left(h_{n}(\theta, z)\right)=-\tau_{c}(n / \ell)^{2}\left(D_{1} h_{n}(0, z)+D_{2} h_{n}(-1, z)\right)+\tau_{c} A h_{n}(0, z) .
$$

By 2.21, we get

$$
\begin{aligned}
& f_{2}^{2}(z, 0,0) \\
= & X_{0}(\theta) \widetilde{F}_{2}\left(\Phi(\theta) z_{x}, 0\right)-\pi\left(X_{0}(\theta) \widetilde{F}_{2}\left(\Phi(\theta) z_{x}, 0\right)\right) \\
= & X_{0}(\theta) \widetilde{F}_{2}\left(\Phi(\theta) z_{x}, 0\right)-\Phi(\theta) \Psi(0)\left(\begin{array}{l}
{\left[\widetilde{F}_{2}\left(\Phi(\theta) z_{x}, 0\right), \beta_{n_{c}}^{(1)}\right]} \\
{\left[\widetilde{F}_{2}\left(\Phi(\theta) z_{x}, 0\right), \beta_{n_{c}}^{(2)}\right]}
\end{array}\right) \gamma_{n_{c}}(x) .
\end{aligned}
$$

By 2.16, 2.40, 2.41) and 2.42, we have

$$
\begin{aligned}
& \left(\begin{array}{l}
{\left[f_{2}^{2}(z, 0,0), \beta_{n}^{(1)}\right]} \\
{\left[f_{2}^{2}(z, 0,0), \beta_{n}^{(2)}\right]}
\end{array}\right) \\
& = \begin{cases}\frac{1}{\sqrt{\ell \pi}} X_{0}(\theta)\left(A_{20} z_{1}^{2}+A_{02} z_{2}^{2}+A_{11} z_{1} z_{2}\right), & n=0, \\
\frac{1}{\sqrt{2 \ell \pi}} X_{0}(\theta)\left(\widetilde{A}_{20} z_{1}^{2}+\widetilde{A}_{02} z_{2}^{2}+\widetilde{A}_{11} z_{1} z_{2}\right), & n=2 n_{c},\end{cases}
\end{aligned}
$$

where $\widetilde{A}_{j_{1} j_{2}}$ is defined as follows

$$
\left\{\begin{array}{l}
\widetilde{A}_{j_{1} j_{2}}=A_{j_{1} j_{2}}-2\left(n_{c} / \ell\right)^{2} A_{j_{1} j_{2}}^{d}, \\
j_{1}, j_{2}=0,1,2, \quad j_{1}+j_{2}=2,
\end{array}\right.
$$

where $A_{j_{1} j_{2}}^{d}$ is determined by 2.43 and $A_{j_{1} j_{2}}$ will be determined in the following. 
Hence, from 2.35), 2.53)-2.55) and matching the coefficients of $z_{1}^{2}, z_{1} z_{2}$, we have

$$
\begin{aligned}
& n=0, \begin{cases}z_{1}^{2}: & \left\{\begin{array}{l}
\dot{h}_{0,20}(\theta)-2 i \omega_{c} h_{0,20}(\theta)=(0,0)^{T}, \\
\dot{h}_{0,20}(0)-L_{0}\left(h_{0,20}(\theta)\right)=\frac{1}{\sqrt{\ell \pi}} A_{20},
\end{array}\right. \\
z_{1} z_{2}:\left\{\begin{array}{l}
\dot{h}_{0,11}(\theta)=(0,0)^{T} \\
\dot{h}_{0,11}(0)-L_{0}\left(h_{0,11}(\theta)\right)=\frac{1}{\sqrt{\ell \pi}} A_{11},
\end{array}\right.\end{cases}
\end{aligned}
$$

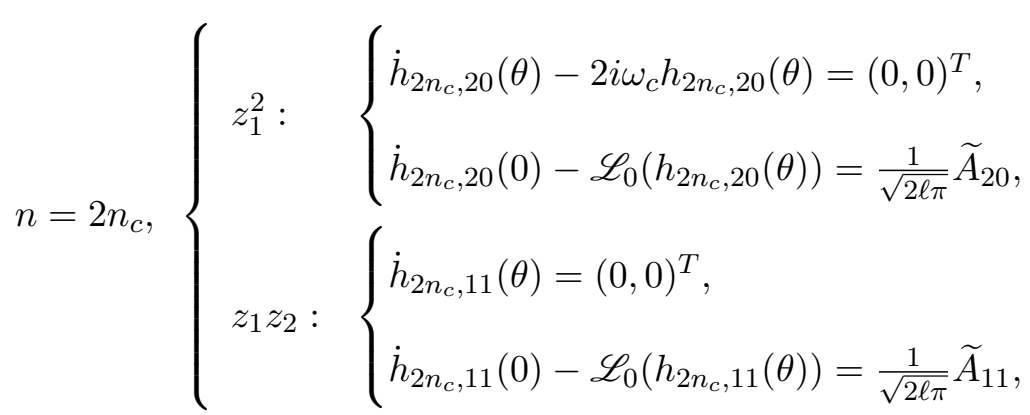

Solving 2.56), 2.57), we obtain

$$
\left\{\begin{array}{l}
h_{0,20}(\theta)=\frac{1}{\sqrt{\ell \pi}}\left(\widetilde{\mathcal{M}}_{0}\left(2 i \omega_{c}\right)\right)^{-1} A_{20} e^{2 i \omega_{c} \theta}, \\
h_{0,11}(\theta)=\frac{1}{\sqrt{\ell \pi}}\left(\widetilde{\mathcal{M}}_{0}(0)\right)^{-1} A_{11}
\end{array}\right.
$$

and

$$
\left\{\begin{array}{l}
h_{2 n_{c}, 20}(\theta)=\frac{1}{\sqrt{2 \ell \pi}}\left(\widetilde{\mathcal{M}}_{2 n_{c}}\left(2 i \omega_{c}\right)\right)^{-1} \widetilde{A}_{20} e^{2 i \omega_{c} \theta} \\
h_{2 n_{c}, 11}(\theta)=\frac{1}{\sqrt{2 \ell \pi}}\left(\widetilde{\mathcal{M}}_{2 n_{c}}(0)\right)^{-1} \widetilde{A}_{11}
\end{array}\right.
$$

where the matrix $\widetilde{\mathcal{M}}_{n}(\lambda)$ is defined by 2.14 .

Remark 2.1. Although the normal form of the delay-induced Hopf bifurcation has extensively studied, the existing procedures can not be applied to the memory-based diffusion system because of the nonlinearity of the diffusion terms and the existence of the delay in the diffusion terms. Compared with the existing procedures, the procedure in our Step 4 is the main characteristic difference.

\section{Application to a predator-prey model with Holling type-II functional response}

We now apply our newly developed algorithm in Section 2 to a a predator-prey model, where in the model (1.1)

$$
f(u, v)=u\left(1-\frac{u}{a}\right)-\frac{b u v}{1+u}, \quad g(u, v)=\frac{b u v}{1+u}-c v .
$$


Then, (1.1) becomes the following predator-prey model with Holling type II functional response:

$$
\begin{cases}\frac{\partial u(x, t)}{\partial t}=d_{11} u_{x x}(x, t)+u\left(1-\frac{u}{a}\right)-\frac{b u v}{1+u}, & 0<x<\ell \pi, t>0, \\ \frac{\partial v(x, t)}{\partial t}=-d_{21}\left(v(x, t) u_{x}(x, t-\tau)\right)_{x}+d_{22} v_{x x}(x, t)-c v+\frac{b u v}{1+u}, & 0<x<\ell \pi, t>0, \\ u_{x}(0, t)=u_{x}(\ell \pi, t)=v_{x}(0, t)=v_{x}(\ell \pi, t)=0, & t \geq 0 .\end{cases}
$$

\subsection{Stability and bifurcation analysis}

System (3.1) has the positive constant steady state $E_{*}\left(u_{*}, v_{*}\right)$, where

$$
u_{*}=\frac{c}{b-c}, \quad v_{*}=\frac{\left(a-u_{*}\right)\left(1+u_{*}\right)}{a b}
$$

provided that $b>\frac{c(1+a)}{a}$ holds. For $E_{*}\left(u_{*}, v_{*}\right)$, we have

$$
\begin{gathered}
a_{11}=\frac{\gamma(a-1-2 \gamma)}{a(1+\gamma)}\left\{\begin{array}{c}
\leq 0, \quad \frac{a-1}{2} \leq \gamma<a, \\
>0, \quad 0<\gamma<\frac{a-1}{2},
\end{array}\right. \\
a_{12}=-c<0, \quad a_{21}=\frac{a-\gamma}{a(1+\gamma)}>0, \quad a_{22}=0,
\end{gathered}
$$

where $\gamma=\frac{c}{b-c}$. Let

$$
J_{n}=d_{11} d_{22}(n / \ell)^{4}-\left(d_{11} a_{22}+d_{22} a_{11}\right)(n / \ell)^{2}+\operatorname{Det}(A),
$$

From $(3.2)$ and $(3.3)$, it is easy to verify that $T_{n}<0$ and $J_{n}>0$ provided that

$$
\left(C_{0}\right) \quad \frac{a-1}{2}<\gamma<a .
$$

This implies that when $d_{21}=0$ and the condition $\left(C_{0}\right)$ holds, the positive steady state $E_{*}$ is asymptotically stable for $d_{11} \geq 0$ and $d_{22} \geq 0$ In what follows, we always assume that the condition $\left(C_{0}\right)$ holds.

Since $J_{n}>0$ under the condition $\left(C_{0}\right)$, we have $\Gamma(0)=J_{n}-d_{21} v_{*} a_{12}>0$. This implies that $\lambda=0$ is not a root of Eq.2.5.

Let $\lambda=i \omega(\omega>0)$ be a root of $(2.5)$. Substituting it along with expressions in 3.2 into (2.5) and separating the real from the imaginary parts, we have

$$
\left\{\begin{array}{l}
\omega^{2}-J_{n}=(n / \ell)^{2} c d_{21} v_{*} \cos (\omega \tau), \\
-T_{n} \omega=(n / \ell)^{2} c d_{21} v_{*} \sin (\omega \tau),
\end{array}\right.
$$

which yields

$$
\omega^{4}+P_{n} \omega^{2}+Q_{n}=0
$$


where

$$
\begin{aligned}
P_{n} & =T_{n}^{2}-2 J_{n} \\
& =\left(d_{11}^{2}+d_{22}^{2}\right)(n / \ell)^{4}-2\left(d_{11} a_{11}+d_{22} a_{22}\right)(n / \ell)^{2}+a_{11}^{2}+a_{22}^{2}+2 a_{12} a_{21} .
\end{aligned}
$$

and

$$
Q_{n}=\left(J_{n}+c d_{21} v_{*}(n / \ell)^{2}\right)\left(J_{n}-c d_{21} v_{*}(n / \ell)^{2}\right) .
$$

and

$$
a_{11}^{2}+a_{22}^{2}+2 a_{12} a_{21} \begin{cases}\leq 0, & c \geq c_{*}, \\ >0, & c<c_{*},\end{cases}
$$

where

$$
c_{*}=\frac{\gamma^{2}(a-1-2 \gamma)^{2}}{2 a(1+\gamma)(a-\gamma)} .
$$

For simplification, we assume that $c<c_{*}$. Then, $P_{n}>0$ for any $n \in \mathbb{N}_{0}$. Define

$$
d_{21}^{(n)}=\frac{J_{n}}{c v_{*}(n / \ell)^{2}}=\frac{1}{c v_{*}}\left(d_{11} d_{22}(n / \ell)^{2}+\frac{\operatorname{Det}(A)}{(n / \ell)^{2}}-\left(d_{11} a_{22}+d_{22} a_{11}\right)\right)>0,
$$

Then, for fixed $n$, by (3.7) we have

$$
Q_{n}\left\{\begin{array}{l}
>0, \quad 0<d_{21}<d_{21}^{(n)} \\
=0, \quad d_{21}=d_{21}^{(n)} \\
<0, \quad d_{21}>d_{21}^{(n)}
\end{array}\right.
$$

Thus, when $d_{21}>d_{21}^{(n)}$, Eq. 3.5 has one positive root $\omega_{n}$, where

$$
\omega_{n}=\sqrt{\frac{-P_{n} \pm \sqrt{P_{n}^{2}-4 Q_{n}}}{2}} .
$$

Notice that $T_{n}<0$. From (3.4), we have

$$
\sin \left(\omega_{n} \tau\right)=\frac{-T_{n} \omega_{n}}{(n / \ell)^{2} c d_{21} v_{*}}>0
$$

Thus, set

$$
\tau_{n, j}=\frac{1}{\omega_{n}}\left\{\arccos \left\{\frac{\omega_{n}^{2}-J_{n}}{d_{21} v_{*} c(n / \ell)^{2}}\right\}+2 j \pi\right\}, \quad j \in \mathbb{N}_{0}, n \in \mathbb{N},
$$

then 2.5 has a pair of purely imaginary roots $\pm \omega_{n} i$ at $\tau=\tau_{n, j}^{+}$. And it is easy to verify the transversality condition satisfies $\left.\frac{d \operatorname{Re}(\lambda(\tau))}{d \tau}\right|_{\tau=\tau_{n, j}}>0$.

Let

$$
d_{21}^{*}=\min _{n \in \mathbb{N}}\left\{d_{21}^{(n)}\right\}>0
$$

From $(3.9)$, it is easy to verify that $d_{21}^{(n)}$ is decreasing for $n<\ell \sqrt[4]{\frac{\operatorname{Det}(A)}{d_{11} d_{22}}}$, is increasing for $n>\ell \sqrt[4]{\frac{\operatorname{Det}(A)}{d_{11} d_{22}}}$ and $d_{21}^{(n)} \rightarrow \infty$ as $n \rightarrow \infty$. This implies that $d_{21}^{*}$ exists. 
For fixed $d_{21}>d_{21}^{*}$, define an index set

$$
U\left(d_{21}\right)=\left\{n \in \mathbb{N}: d_{21}^{(n)}<d_{21}\right\} .
$$

By the above discussion, we obtain the following results on the stability and Hopf bifurcation of system (3.1).

Theorem 3.1. Assume that the condition $\left(C_{0}\right)$ holds and $c<c_{*}$. Then we have the following:

(a) when $0<d_{21} \leq d_{21}^{*}$, the positive constant steady state $\left(u_{*}, v_{*}\right)$ of system (3.1) is locally asymptotically stable for any $\tau \geq 0$;

(b) when $d_{21}>d_{21}^{*}$, there exists a critical value $\tau_{*}\left(d_{21}\right)$ of the delay such that

(b1) the positive constant steady state $\left(u_{*}, v_{*}\right)$ of system (3.1) is locally asymptotically stable for $0 \leq \tau<\tau_{*}\left(d_{21}\right)$ and unstable for $\tau>\tau_{*}\left(d_{21}\right)$, where

$$
\tau_{*}\left(d_{21}\right)=\min _{n \in U\left(d_{21}\right)}\left\{\tau_{n, 0}\right\} .
$$

(b2) system (3.1) undergoes mode-n Hopf bifurcation at $\tau=\tau_{n, j}, n \in U\left(d_{21}\right)$, and multiple Hopf bifurcations occur when these Hopf bifurcation curves $\tau=\tau_{n, j}, n \in U\left(d_{21}\right)$ have interaction point on the $d_{21}-\tau$ plane.

\subsection{Direction and stability of the Hopf bifurcation}

We now numerically investigate the bifurcation direction and stability of the bifurcation. To this end, we set the parameters as follows

$$
a=1, b=\frac{3}{10}, c=\frac{1}{10}, d_{11}=\frac{3}{5}, d_{22}=\frac{4}{5}, \ell=2 .
$$

Then, we have $\left(u_{*}, v_{*}\right)=(1 / 5,5 / 2), \gamma=1 / 2$,

$$
a_{11}=-\frac{1}{3}, a_{12}=-\frac{1}{10}, a_{21}=\frac{1}{3}, a_{22}=0
$$

and $\operatorname{Tr}(A)=-1 / 2, \operatorname{Det}(A)=1 / 30$. It follows from $(3.6)$ and $(3.9)$ that

$$
P_{n}=\frac{1}{16} n^{4}+\frac{1}{10} n^{2}+\frac{2}{45}>0
$$

and

$$
d_{21}^{(n)}=\frac{12 n^{2}}{25}+\frac{8}{15 n^{2}}+\frac{16}{15} .
$$

Notice that $P_{n}>0$ for any $n \in \mathbb{N}$, which together with (3.10), implies that for a fixed $n$, Eq. 3.5 has no positive root for $d_{21}<d_{21}^{(n)}$ and has only one positive root for $d_{21} \geq d_{21}^{(n)}$. From (3.14), it is easy to verify that $d_{21}^{(n)}<d_{21}^{(n+1)}$ for any $n \in \mathbb{N}$, and

$$
d_{21}^{(1)}=2.08<d_{21}^{(2)}=3.12<d_{21}^{(3)} \doteq 5.4459 .
$$




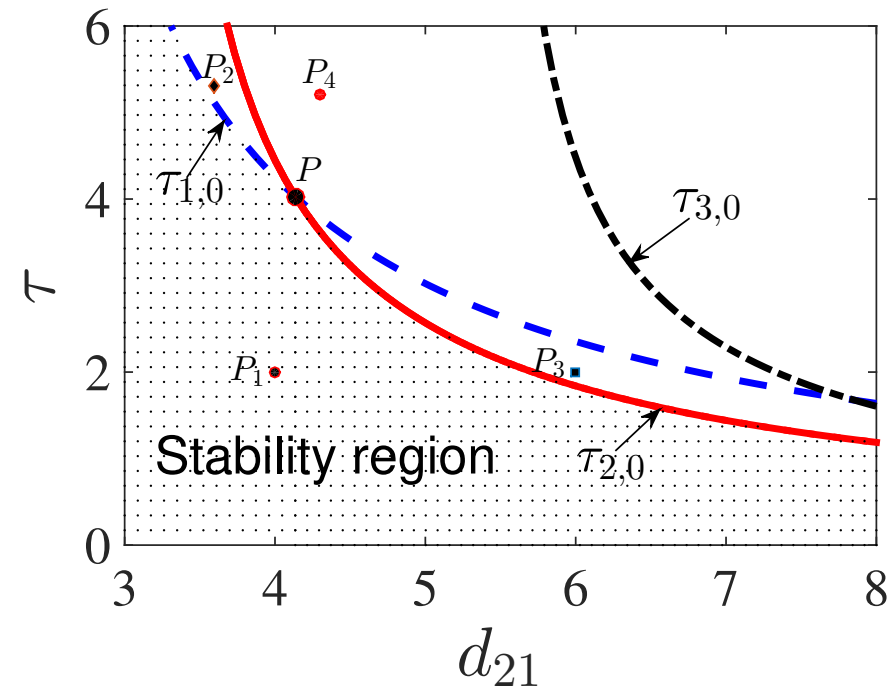

Figure 1: Stability region and Hopf bifurcation curves in $d_{21}-\tau$ plane. The dotted region is the stability region and $\tau=\tau_{k, 0}, k=1,2,3$, are Hopf bifurcation curves. Hopf bifurcation curves $\tau=\tau_{1,0}$ and $\tau=\tau_{2,0}$ intersect at the point $P(4.1354,4.0292)$. The points $P_{1}(4,2), P_{2}(3.6,5.3)$, $P_{3}(6,2)$ and $P_{4}(4.3,5.2)$ are chosen for the numerical simulations.

Therefore, by 3.13 , we have $d_{21}^{*}=d_{21}^{(1)}=2.08$. It follows from 3.8 that $c_{*}=1 / 6$. By Theorem (3.1), we have the following stability result.

Proposition 3.2. For system (3.1) with the parameters $a=1, b=\frac{3}{10}, c=\frac{1}{10}, d_{11}=\frac{6}{10}, d_{22}=$ $\frac{8}{10}$, $\ell=2$, when $0 \leq d_{21}<d_{21}^{(1)}=2.08$, the positive constant steady state $\left(u_{*}, v_{*}\right)=(1 / 5,5 / 2)$ is locally asymptotically stable for any $\tau \geq 0$;

For fixed $d_{21}>d_{21}^{(1)}$, the positive constant steady state $\left(u_{*}, v_{*}\right)$ is asymptotically stable for $\tau<\tau^{*}\left(d_{21}\right)$ and unstable for $\tau>\tau^{*}\left(d_{21}\right)$. Fig 1 illustrates the stability region and the Hopf bifurcation curves in the $d_{21}-\tau$ plane for $3 \leq d_{21} \leq 8$ and $0 \leq \tau \leq 6$. The Hopf bifurcation curves $\tau=\tau_{1,0}$ and $\tau=\tau_{2,0}$ intersect at the point $P_{1}(4.1354,4.0292)$, which is the double Hopf bifurcation point. For the point $P_{1}(4,2)$ located in the stability region, Fig.2 illustrates the evolution of the solution of system (3.1) starting from the initial values $u_{0}(x)=$ $0.2+0.1 \cos (x / 2), v_{0}(x)=2.5+0.1 \cos (x / 2)$, finally converging to the constant steady state $\left(u_{*}, v_{*}\right)$.

From Fig, 1, it is obvious to see that

$$
\tau^{*}\left(d_{21}\right)= \begin{cases}\tau_{1,0}, & d_{21}^{(1)}<d_{21}<4.1354 \\ \tau_{2,0}, & 4.1354<d_{21}<8\end{cases}
$$



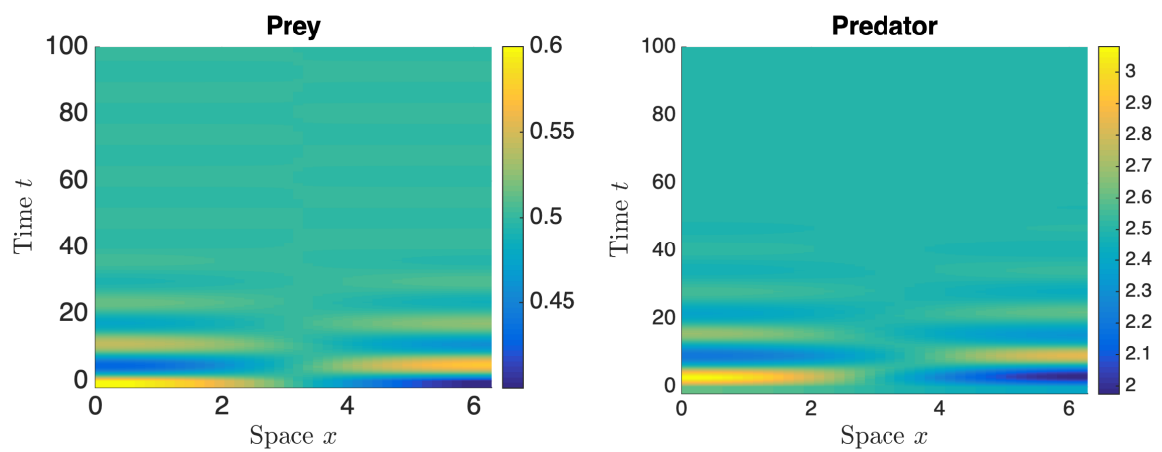

Figure 2: The spatial-temporal dynamics of system (3.1) with the parameters $a=1, b=$ $\frac{3}{10}, c=\frac{1}{10}, d_{11}=\frac{6}{10}, d_{22}=\frac{8}{10}, \ell=2$ and $\left(d_{21}, \tau\right)$ being chosen as the point $P_{1}(4,2)$ of Fig 1. The constant steady state is stable is asymptotically stable. The initial values are $u_{0}(x)=0.2+0.1 \cos (x / 2), v_{0}(x)=2.5+0.1 \cos (x / 2)$.

For $d_{21}=3.6$, it follows from 3.11 and 3.12 that

$$
\tau_{1,0} \doteq 5.1033<\tau_{2,0} \doteq 6.6493
$$

For the critical mode- 1 Hopf bifurcation at $\tau_{1,0} \doteq 5.1033$, the direction and stability of this Hopf bifurcation can be determined by the procedure in the previous section with $\tau_{c}=\tau_{1,0} \doteq 5.1033$ and $d_{21}^{c}=3.6$. A directional calculation shows that

$$
K_{1} \doteq 0.0597>0, \quad K_{2} \doteq-1.5624<0,
$$

which implies that the mode-1 spatially imhomogeneous Hopf bifurcation at $\tau_{1,0}$ is supercritical and stable. For $\tau=5.2>\tau_{1,0}$, Fig $\sqrt[3]{3(a)}-(b)$ illustrate the existence of the spatially inhomogeneous periodic solution with mode-1 spatial pattern.

For $d_{21}=6$, it follows from (3.11) and $(3.12)$ that

$$
\tau_{2,0} \doteq 1.8398<\tau_{1,0} \doteq 2.3542
$$

For the critical Hopf bifurcation value $\tau_{2,0} \doteq 1.8398$, we have

$$
K_{1} \doteq 0.1733>0, \quad K_{2} \doteq-2.2283<0,
$$

which implies that the mode-2 spatially imhomogeneous Hopf bifurcation at $\tau_{2,0}$ is also supercritical and stable. For $\tau=2>\tau_{2,0}$, Fig. $3(c)-(d)$ illustrate the existence of the spatially inhomogeneous periodic solution with mode-2 spatial pattern.

We would like to mention that the interaction of mode- 1 and mode-2 Hopf bifurcations leads to more complex dynamics. To investigate the dynamical classification near the double Hopf point $P$ in detail, the normal form for double Hopf bifurcation should be further developed. For 


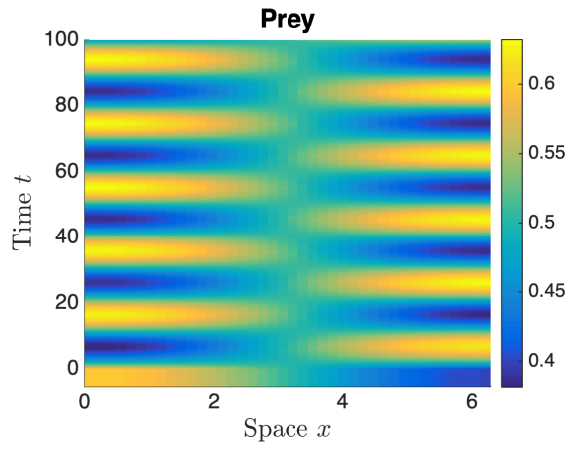

(a)

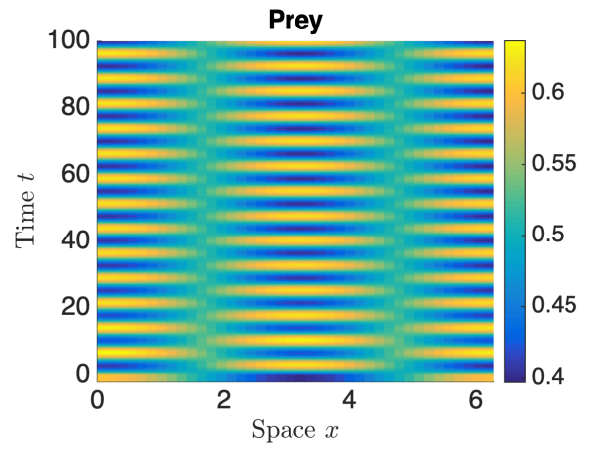

(c)

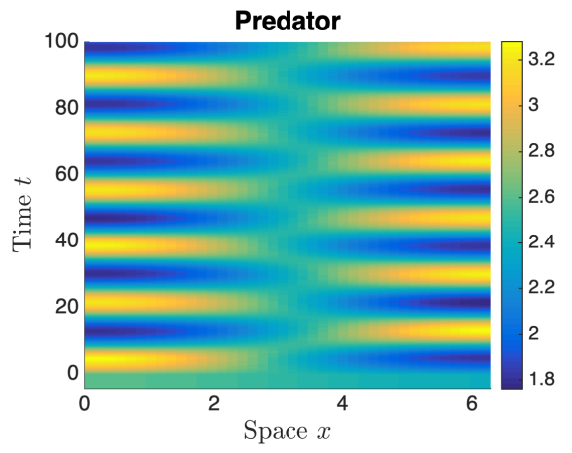

(b)

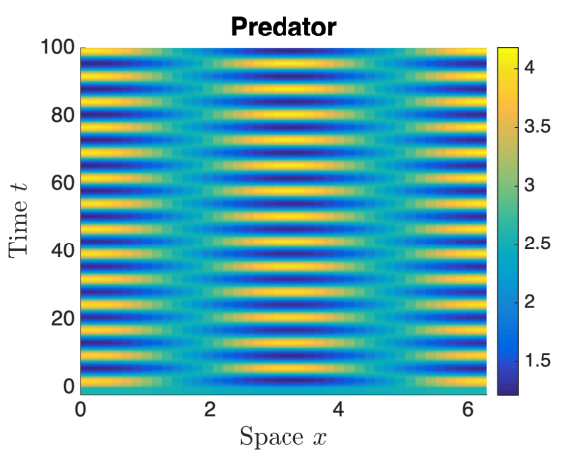

(d)

Figure 3: The spatial-temporal dynamics of system (3.1) with the parameters $a=1, b=$ $\frac{3}{10}, c=\frac{1}{10}, d_{11}=\frac{6}{10}, d_{22}=\frac{8}{10}, \ell=2 .(a)-(b)$ For the point $P_{2}(3.6,5.3)$ of Fig 1 , there exists a spatially inhomogeneous periodic solution with mode-1 spatial pattern. The initial values are $u_{0}(x)=0.2+0.1 \cos (x / 2), v_{0}(x)=2.5+0.1 \cos (x / 2) .(c)-(d)$ For the point $P_{3}(6,2)$ of Fig. 1. there exists a spatially inhomogeneous periodic solution with mode-2 spatial pattern. The initial values are $u_{0}(x)=0.2+0.1 \cos (x), v_{0}(x)=2.5+0.1 \cos (x)$. 

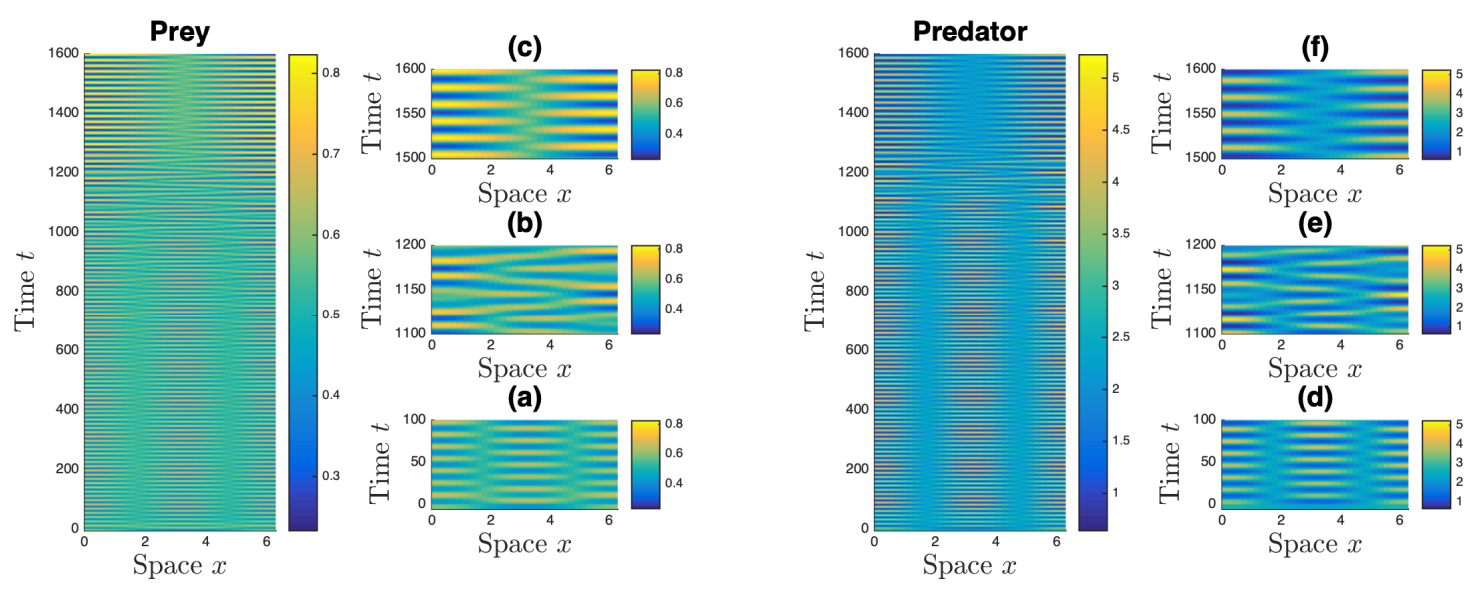

Figure 4: The spatial-temporal dynamics of system (3.1) with the parameters $a=1, b=$ $\frac{3}{10}, c=\frac{1}{10}, d_{11}=\frac{6}{10}, d_{22}=\frac{8}{10}, \ell=2$ and $\left(d_{21}, \tau\right)$ being the point $P_{4}(4.3,5.2)$ of Fig 1 far from the Hopf bifurcation curves. Pattern transition from the mode-2 spatially inhomogeneous periodic solution to mode- 1 spatially inhomogeneous periodic solution. The initial values are $u_{0}(x)=0.2+0.1 \cos (x), v_{0}(x)=2.5+0.2 \cos (x)$.

the point $P_{4}(4.3,5.2)$ of Fig. 1 far from the Hopf bifurcation curves $\tau=\tau_{k, 0}, k=1,2$, Fig 4 shows the pattern transition from the mode-2 spatially inhomogeneous periodic solution (Figs $4(a)$ and $(d)$ ) to mode-1 spatially inhomogeneous periodic solution (Figs $4(c)$ and $(f))$. Figs $4(b)$ and $(e)$ also illustrate the transiently unstable quasi-periodic patterns.

\section{Conclusion and discussion}

In this paper, we have developed an algorithm for computing the normal form of the Hopf bifurcation for the reaction-diffusion systems with memory diffusion. Because of the nonlinearity of the diffusion term and the the presence of the delay in the diffusion term, the traditional algorithms for computing the normal form of the Hopf bifurcation for the reaction-diffusion system is not applied to this system. To fill this gap, we generalized the existing algorithms for the reaction-diffusion systems where the diffusion terms are linear and the delay only appears in the reaction terms, to the case where the diffusion terms are not linear and the delay appears not only in the reaction terms but also in the diffusion terms.

As an illustration of this newly developed algorithm, we considered a diffusive predatorprey system with memory-based diffusion and Holling type-II functional response. The memory delay-induced spatially inhomogeneous Hopf bifurcations and the double Hopf bifurcations due to their interactions are observed.

We would also like to mention that the delay-induced double Hopf bifurcation often occurs 
in reaction-diffusion systems with memory diffusion and may lead to more complex dynamics like two/three invariant torus. To determine the dynamical classifications near the double Hopf bifurcation points, new algorithms for computing the normal form of the double Hopf bifurcation of the reaction-diffusion systems with memory diffusion are desired.

\section{References}

[1] Qi An, Chuncheng Wang, and Hao Wang. Analysis of a spatial memory model with nonlocal maturation delay and hostile boundary condition. Discret. Contin. Dyn. Syst., 40(10):58455868, OCT 2020.

[2] Jianxin Chen, Tonghua Zhang, and Yongwu Zhou. Dynamics of a risk-averse newsvendor model with continuous-time delay in supply chain financing. Math. Comput. Simul., 169:133-148, MAR 2020.

[3] Mengxin Chen, Ranchao Wu, Biao Liu, and Liping Chen. Spatiotemporal dynamics in a ratio-dependent predator-prey model with time delay near the Turing-Hopf bifurcation point. Commun. Nonlinear Sci. Numer. Simul., 77:141-167, OCT 2019.

[4] S-N Chow and J K Hale. Methods of Bifurcation Theory. Springer-Verlag, New York, 1982.

[5] Huan Dai, Yuying Liu, and Junjie Wei. Stability analysis and Hopf bifurcation in a diffusive epidemic model with two delays. Math. Biosci. Eng., 17(4):4127-4146, 2020.

[6] T Faria. Normal forms and Hopf bifurcation for partial differential equations with delays. Trans. Am. Math. Soc., 352(5):2217-2238, 2000.

[7] T Faria and L T Magalhães. Normal forms for retarded functional differential equations with parameters and applications to Hopf singularity. J. Differ. Equ., 122(2):181-200, NOV 11995.

[8] Jack K Hale. Theory of Functional Differential Equations. Springer-Verlag, Berlin, 1977.

[9] B.D. Hassard, N.D. Kazarinoff, and Y.H. Wan. Theory and Applications of Hopf Bifurcation. Cambridge University Press, Cambridge, 1981.

[10] Weihua Jiang, Hongbin Wang, and Xun Cao. Turing instability and Turing-Hopf bifurcation in diffusive Schnakenberg systems with gene expression time delay. J. Dyn. Differ. Equ., 31(4):2223-2247, DEC 2019. 
[11] Xin Jiang, Ran Zhang, and Zhikun She. Dynamics of a diffusive predator-prey system with ratio-dependent functional response and time delay. Int. J. Biomath., 13(6):2050036, AUG 2020.

[12] Longyue Li, Yingying Mei, and Jianzhi Cao. Hopf Bifurcation Analysis and Stability for a Ratio-Dependent Predator-Prey Diffusive System with Time Delay. Int. J. Bifurcation Chaos, 30(3):2050037, MAR 152020.

[13] Jiazhe Lin, Rui Xu, and Liangchen Li. Turing-Hopf bifurcation of reaction -diffusion neural networks with leakage delay. Commun. Nonlinear Sci. Numer. Simul., 85:105241, JUN 2020.

[14] Kalyan Manna and Malay Banerjee. Stability of Hopf-bifurcating limit cycles in a diffusion-driven prey-predator system with Allee effect and time delay. Math. Biosci. Eng., 16(4):2411-2446, 2019.

[15] Xin-You Meng and Jiao-Guo Wang. Dynamical analysis of a delayed diffusive predator-prey model with schooling behaviour and Allee effect. J. Biol. Dyn., 14(1):826-848, JAN 12020.

[16] Karen A. Oliveira and Juliana M. Berbert. Crossover in spreading behavior due to memory in population dynamics. Math. Biosci., 324:108346, JUN 2020.

[17] Zuolin Shen and Junjie Wei. Bifurcation Analysis in a Diffusive Mussel-Algae Model with Delay. Int. J. Bifurcation Chaos, 29(11): 10.1142/S021812741950144X, OCT 2019.

[18] Junping Shi, Chuncheng Wang, and Hao Wang. Diffusive spatial movement with memory and maturation delays. Nonlinearity, 32(9):3188-3208, SEP 2019.

[19] Junping Shi, Chuncheng Wang, Hao Wang, and Xiangping Yan. Diffusive Spatial Movement with Memory. J. Dyn. Differ. Equ., 32(2):979-1002, JUN 2020.

[20] Qingyan Shi, Junping Shi, and Hao Wang. Spatial movement with distributed delay. J. Math. Biol., 28(4):https://doi.org/10.1007/s00285-021-01588-0, 2021.

[21] Y. Song, H. Jiang, and Y. Yuan. Turing-Hopf bifurcation in the reaction-diffusion system with delay and application to a diffusive predator-prey model. J. Appl. Anal. Comput., 9(3):1132-1164, JUN 2019.

[22] Yongli Song, Junping Shi, and Hao Wang. Spatiotemporal dynamics of a diffusive consumerresource model with explicit spatial memory. , page submitted for review, 2021. 
[23] Yongli Song and Qingyan Shi. Stability and spatially non-homogeneous Hopf bifurcation in a diffusive delayed predator-prey model with spatial average. , page submitted for review, 2020.

[24] Yongli Song, Shuhao Wu, and Hao Wang. Spatiotemporal dynamics in the single population model with memory-based diffusion and nonlocal effect. J. Differ. Equ., 267(11):6316-6351, NOV 152019.

[25] Yongli Song, Tonghua Zhang, and Yahong Peng. Turing-Hopf bifurcation in the reactiondiffusion equations and its applications. Commun. Nonlinear Sci. Numer. Simul., 33:229258, APR 2016.

[26] Xiaosong Tang, Heping Jiang, Zhiyun Deng, and Tao Yu. Delay induced subcritical Hopf bifurcation in a diffusive predator-prey model with herb behavior and hyperbolic mortality . J. Appl. Anal. Comput., 7(4):1385-1401, 2017.

[27] Xiaosong Tang and Yongli Song. Stability, Hopf bifurcations and spatial patterns in a delayed diffusive predator-prey model with herd behavior. Appl. Math. Comput., 254:375391, MAR 12015.

[28] Shuhao Wu and Yongli Song. Spatiotemporal dynamics of a diffusive predator-prey model with nonlocal effect and delay. Commun. Nonlinear Sci. Numer. Simul., 89:105310, OCT 2020.

[29] Ruizhi Yang, Ming Liu, and Chunrui Zhang. A delayed-diffusive predator-prey model with a ratio-dependent functional response. Commun. Nonlinear Sci. Numer. Simul., 53:94-110, DEC 2017.

[30] Ruizhi Yang, Chunrui Zhang, and Yazhuo Zhang. A delayed diffusive predator-prey system with Michaelis-Menten type predator harvesting. Int. J. Bifurcation Chaos, 28(8):1850099, JUL 2018.

[31] Jia-Fang Zhang and Xiang-Ping Yan. Effects of Delay and Diffusion on the Dynamics of a Leslie-Gower Type Predator-Prey Model. Int. J. Bifurcation Chaos, 24(4):1450043, APR 2014.

[32] Wenjie Zuo and Junjie Wei. Stability and Hopf bifurcation in a diffusive predator-prey system with delay effect. Nonlinear Anal.-Real World Appl., 12(4):1998-2011, AUG 2011.

[33] Wenjie Zuo and Junjie Wei. Stability and bifurcation in a ratio-dependent Holling-III system with diffusion and delay. Nonlinear Anal.-Model Control, 19(1):132-153, 2014. 